\title{
Evaluating Acoustical Performance Of Existing Offices As Part Of A WELL Feasibility Service
}

\author{
Ryan Bessey *, Jessie Roy ${ }^{\dagger}$ \\ RWDI 901 King Street West, Suite 400, Toronto, Ontario
}

\section{Introduction}

While the WELL building standard is typically applied during the design of new offices, it can also be applied retroactively to existing offices. Before undertaking such an endeavour, companies may be interested in evaluating their office's acoustical performance with respect to the standard. This paper describes how a WELL feasibility assessment can be performed for the acoustically-focused WELL features. This allows companies to know where they stand with respect to the WELL building standard and what it might take to achieve the different levels of certification.

\subsection{Versions of the WELL standard}

The WELL standard is currently at v2.0, which brought many changes from v1.0 upon which this feasibility service was initially based. The content has been updates considering these changes. Note that v2.0 is currently in Beta testing; project team can choose to pursue certification under v1.0 or v2.0; and may apply to use v2.0 features for projects registered under v1.0.

In the new version, all acoustically-focused "Features" have been moved into a separate "Sound" category with aspects related to background noise levels, sound isolation, privacy, speech intelligibility and room acoustics. Each of these will be discussed with respect to determining feasibility.

\subsection{Feasibility assessment approach}

The approach for the feasibility assessment is to minimize disruption to an occupied office and the amount of effort required on-site. Therefore, many aspects of the assessment are based on pre-site visit drawing reviews followed by visual inspections on site. Measurements are limited to background sound levels and balloon-pop reverberation time measurements since these are relatively unobtrusive compared to sound isolation measurements.

\section{WELL v2.0 acoustical features}

The WELL v2.0 Standard has 5 features related to acoustics, which will be discussed with respect to performing a feasibility assessment.

\footnotetext{
ryan.bessey@rwdi.com

$\dagger$ jessie.roy@rwdi.com
}

\subsection{S01 - Sound mapping}

Achieving the "Sound Mapping" feature is critical since it is a Pre-Condition for WELL certification. The purpose of this feature is to provide the basic components of acoustically comfortable and productive workspaces. The requirements of this feature are related to background sound levels from both HVAC and exterior noise sources, acoustical privacy, and adequate separation of loud and quiet areas.

In assessing background sound, it is simple to perform a few spot check measurements in an occupied office to get a general sense of HVAC noise levels and exterior noise intrusion such as from road and rail traffic. While with v1.0 of the standard, these had to be measured separately, with v2.0 they are now combined into a single assessment. It is therefore possible to measure both exterior noise and interior HVAC noise simultaneously. That said, one source of noise that should not be included is that of people talking. For a quiet office, this may not be much of an issue but for an office with a lot of chatter this could require early morning or evening measurements. A good approach is to take several spot checks at various locations around the office noting the dominant noise source during each measurement. This will allow for efficient data screening.

While this feature does have requirements related to sound isolation and acoustical privacy, there are no specific targets. All that seems to be required is to document expected or measured sound isolation performance.

In the final part of the Sound Mapping feature, it is required to produce a drawing with acoustic zones labelled. Presumably the intention is to show that space planning considered acoustics before the office was built, but for an existing office it may still be useful to apply Loud, Quiet, and Mixed labels to different zones to influence employee behaviors.

\subsection{S02 - Maximum noise levels}

This feature contains one part called "Limited Background Noise Levels" with suggested $\mathrm{dBA}$ and $\mathrm{dBC}$ targets based on the both $\mathrm{L}_{\mathrm{eq}}$ and $\mathrm{L}_{\mathrm{Max}}$ metrics. Since this is an Optimization, points are awarded for achieving different target ranges. Since the targets are based on different weightings and metrics, it's useful to have a Sound Level Meter (SLM) that can measure everything simultaneously. Combined with a SLM capable of statistics and/or logging, this allows measurements to be performed while walking through an office. 


\subsection{S03 - Sound barriers}

A more difficult aspect to assess quantitatively in an occupied office is sound isolation and privacy. However, since this is an Optimization, it is not required, but points are awarded for achieving different target ranges.

Part 1 of the "Sound Barriers" feature requires minimum Speech Privacy Potential (SPP) targets to be achieved for different room types. While a drawing review can give a general sense of a walls expected sound isolation performance, this does not factor in construction quality. Qualitatively during a site visit it is possible to visually inspect for sound isolation issues such as walls that do not extend above ceiling tiles, gaps where partitions meet, unsealed doors, etc. It is also possible to listen for sound isolation issues. For example, if someone is talking outside a room and you can clearly hear them. While not as conclusive as a quantitative assessment, apparent sound isolation issues can be flagged as potentially problematic. Based on estimates of sound isolation performance and measured background sound levels, the SPP can be determined for comparison against the target ranges.

Part 2 of this feature is related to the sound isolation performance of entrance doors. Since this part does not require measurements, qualification can easily be determined by inspecting the doors seals and construction.

\subsection{S04 - Sound Absorption}

Sound absorption is related to speech intelligibility, privacy and noise build-up due to reverberation. Since this is an Optimization, it is not required, but points are awarded for achieving different target ranges.

Part 1 of the "Sound Absorption" feature requires maximum Reverberation Time (RT) targets to be achieved for different room types. These can be measured relatively quickly on site using a balloon pop test, or estimated based on RT spreadsheet models. These should give a general sense of whether the office qualifies for the available point.

Part 2 and 3 are related to the acoustical ceiling tile and acoustical wall treatments for certain rooms, which can easily be determined from percentage area estimates and review of product specifications.

\subsection{S05 - Sound Masking}

Sound masking is related to privacy and helps minimize distractions. Since this is an Optimization, it is not required, but points are awarded if a sound masking system is installed and operates in the target sound level ranges.

Whether an office meets the requirements of this feature can easily be determined by visual inspection of the ceiling or ceiling plenum accompanied by spot-check sound level measurements.

\section{Conclusion}

Based on a combination of visual inspections and spotcheck sound level measurements, it is possible assess the feasibility of compliance for an existing office with the acoustical features of the WELL building standard. While "Sound" is just one category of the WELL standard, it is also possible to assess compliance with the other aspects of the standard through visual inspects, spot-check measurements, and unattended monitoring. Thus, a full assessment with respect to the WELL Standard can be performed for clients interested in having their offices evaluated.

\section{Acknowledgments}

The authors would like to thank Sandra Dedesko and Mike Williams from RWDI and Michelle Xuereb and Vera Gisarov from Quadrangle Architects for their help in developing this feasibility assessment.

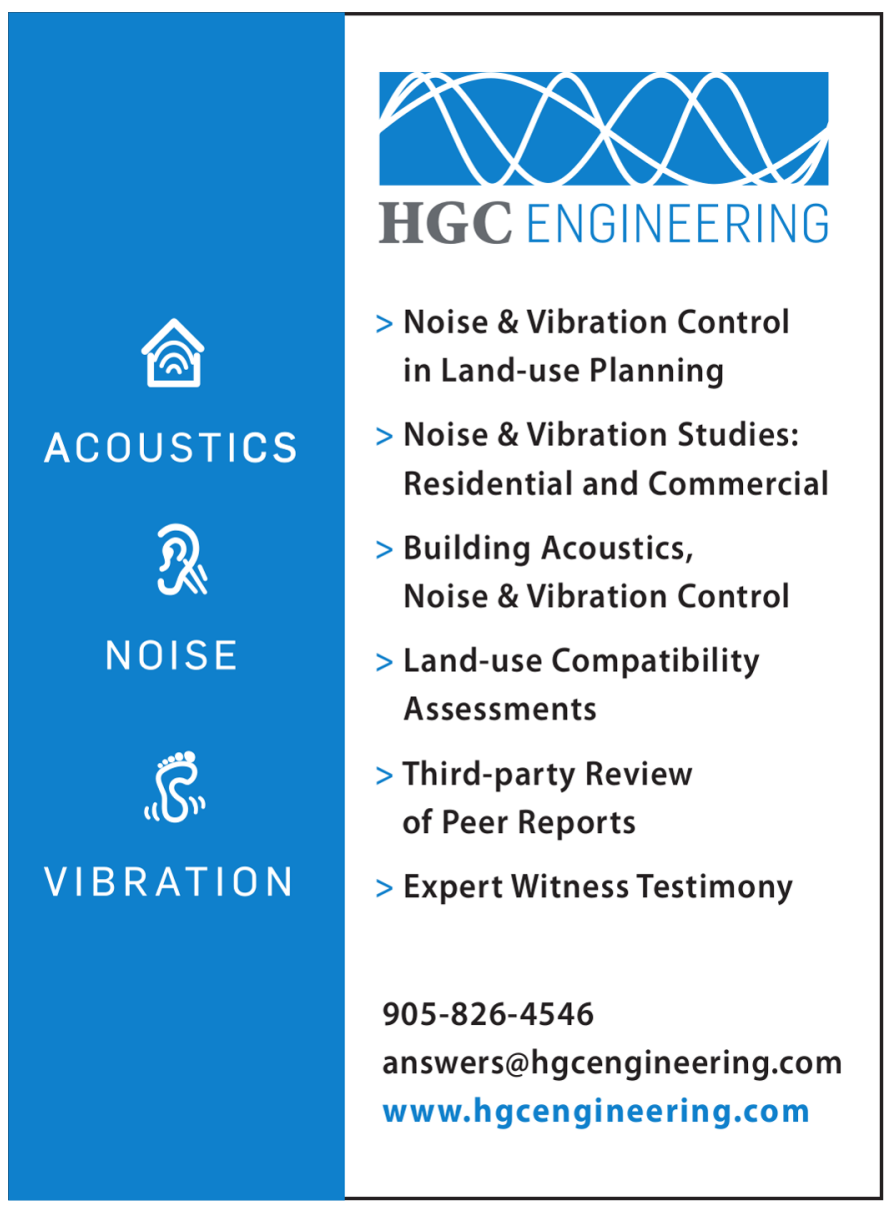



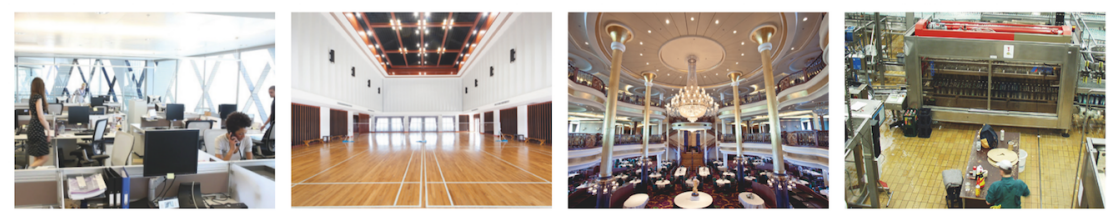

Cadna D) $\mathbf{R}^{n}$

Noise Levels inside Rooms

\section{CadnaR is the powerful software for the calculation and assessment of sound levels in rooms and at workplaces}

\section{:- Intuitive Handling}

The clearly arranged software enables the user to easily build models and make precise predictions. At the same time you benefit from the sophisticated input possibilities as your analysis becomes more complex.

\section{:- Efficient Workflow}

Change your view from 2D to 3D within a second. Multiply the modeling speed by using various shortcuts and automation techniques. Many time-saving acceleration procedures enable a fast calculation process.

\section{:- Modern Analysis}

CadnaR uses scientific and highly efficient calculation methods. Techniques like scenario analysis, grid arithmetic or the display of results within a 3D-grid enhance your analysis and support you during the whole planning and assessment process.
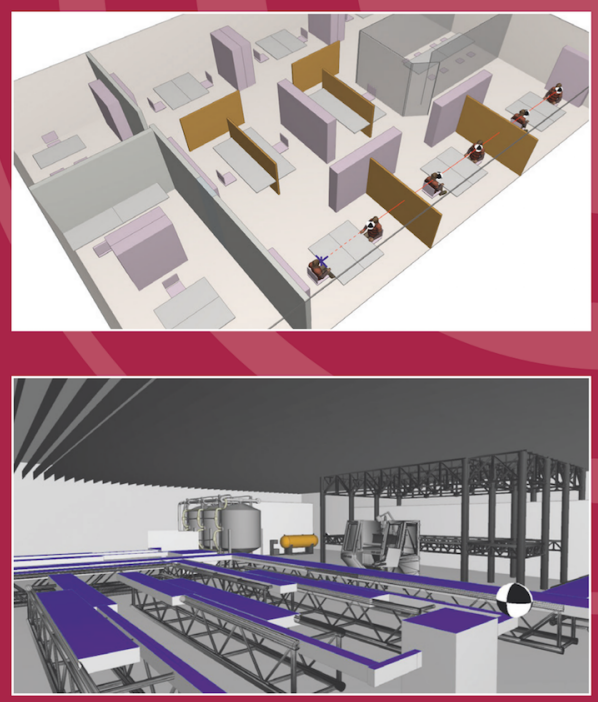

\section{Fields of Application}

Office Environments

- Process your acoustic calculations and assessments according to DIN 18041, VDI 2569 and ISO 3382-3

- Receiver chains serve as digital "measurement path" and provide you with relevant insights into the acoustic quality of rooms during the planning phase

- Import of DWG-/DXF-/SKP-files (e.g. pCon.planner, AutoCAD, SketchUp)

- Visualization of noise propagation, noise levels and parameters for quality criteria like the Speech Transmission Index STI

Production Plants

- Calculation of the sound load at workplaces based on the emission parameters specified by the machine manufacturer according to the EC guideline 2006/42/EC while also taking the room geometry and the room design into account

- Tools for enveloping surfaces and free field simulations to verify the sound power of the sources inside of the enveloping surface

- Calculation of the sound power level based on technical parameters such as rotational speed or power

\section{D) DataKustik}

Distributed in the U.S. and Canada by: Scantek, Inc. Sound and Vibration Instrumentation and Engineering 6430 Dobbin Rd, Suite C | Columbia, MD 21045 | 410-290-7726 | www.scantekinc.com 


\section{0 \\ $\overline{\text { ANNIVERSARY }}$ \\ McoustiGuard’ WILREP LTD.}

\section{SOUND and VIBRATION CONTROL}

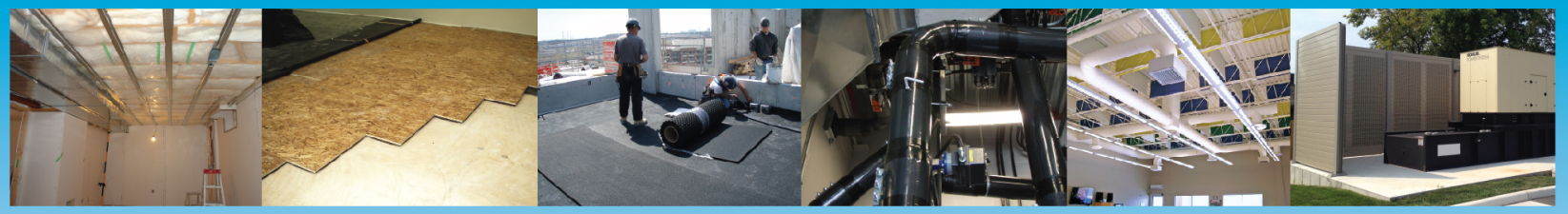

Since 1977, AcoustiGuard - WILREP LTD. has been providing products and solutions for sound and vibration control.

We are now pleased to announce the addition of our new ARCHITECTURAL ACOUSTICS product line.

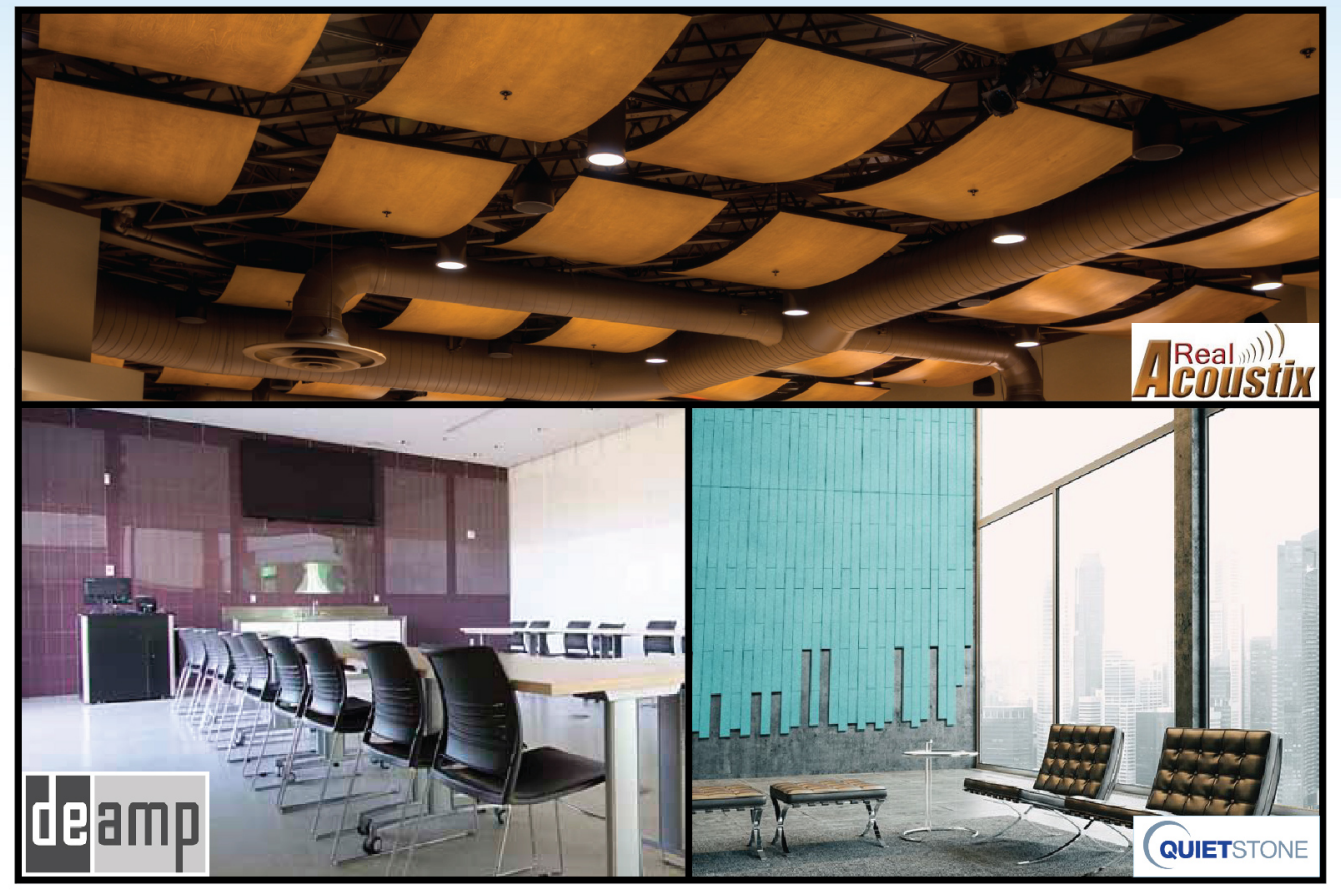

Exclusive Canadian Dealer for RealAcoustix Exclusive North American Distribution of DeAmp and QuietStone www.acoustiguard.com 1-888-625-8944 